\title{
Turbulence structure of open channel flows over permeable and impermeable beds: A comparative study
}

\author{
Costantino Manes, ${ }^{1, a)}$ Dubravka Pokrajac, ${ }^{2}$ Ian McEwan, ${ }^{2}$ and Vladimir Nikora ${ }^{2}$ \\ ${ }^{1}$ Dipartimento di Idraulica, Trasporti ed Infrastrutture Civili (DITIC), Politecnico di Torino, \\ Corso Duca degli Abruzzi, Torino 10129, Italy \\ ${ }^{2}$ Department of Engineering, Fraser Noble Building, Kings College, Aberdeen AB243UE, \\ Scotland, United Kingdom
}

(Received 12 June 2009; accepted 13 November 2009; published online 31 December 2009)

\begin{abstract}
The behavior of turbulent open channel flows over permeable surfaces is not well understood. In particular, it is not clear how the surface and the subsurface flow within the permeable bed interact and influence each other. In order to clarify this issue we carried out two sets of experiments, one involving velocity measurements in open channel flows over an impermeable bed composed of a single layer of spheres, and another one where velocities were measured over and within a permeable bed made of five such layers. Comparison of surface flow velocity statistics between the two sets of experiments confirmed that bed permeability can significantly affect flow resistance. It was also confirmed that even in the hydraulically rough regime, the friction factors for the permeable bed increase with increasing Reynolds number. Such an increase in flow resistance implies a different distribution of normal form-induced stress between the permeable and impermeable bed cases. Subsurface flow measurements performed within the permeable bed revealed that there is an intense transport of turbulent kinetic energy (TKE) occurring from the surface to the subsurface flow. We provide evidence that the transport of TKE toward the lower bed levels is driven mainly by pressure fluctuations, whereas TKE transport due to turbulent velocity fluctuations is limited to a thinner layer placed in the upper part of the bed. It was also confirmed that the turbulence imposed by the surface flow gradually dissipates while penetrating within the porous medium. Dissipation occurs faster for the small scales than for the large ones, which instead are persistent, although weak, even at the lowest bed levels. (C) 2009 American Institute of Physics. [doi:10.1063/1.3276292]
\end{abstract}

\section{INTRODUCTION}

In general, the domain of an open channel flow over a porous bed can be divided into surface and subsurface flow regions, which are located above and within the bed, respectively (Fig. 1). The transition layer is defined as that portion of the subsurface flow which is influenced by the turbulent motion of the surface flow (Fig. 1). The hydrodynamic processes that control the interaction between surface and subsurface flows play an important role in the functioning of stream ecosystems and ecologically relevant mass transfer processes occurring at the sediment water interface of natural streams (i.e., the so called hyporheic exchange processes). Furthermore, the generic problem of flows over permeable walls is relevant for a range of environmental and industrial applications such as urban buildings, coastal defense, and heat exchangers.

Despite its importance, the behaviors of turbulent open channel flows over permeable surfaces have not been sufficiently investigated and many issues concerning the interaction between surface and subsurface flows need to be elucidated. In particular, it is not clear how the permeability of the bed influences the turbulence properties of the surface flow and in turn, how the surface flow interacts with the subsur-

\footnotetext{
a) Author to whom correspondence should be addressed. Electronic mail: costantino.manes@polito.it.
}

face flow within the transition layer. Sections I A-I C present a review of the current knowledge on these two topics, highlight the associated shortcomings, and outline the contribution from the present paper.

\section{A. The effects of permeability on the surface flow}

In many engineering applications, permeability is usually neglected and turbulent flows over porous beds are considered equivalent to standard rough-wall turbulent boundary layers over impermeable beds. However, there is a substantial difference between flows over impermeable and permeable beds. In the former case, the no-slip condition applies to the solid surface whereas in the latter case, the flow is able to penetrate into the bed and to interact with the interstitial fluid. Such interaction includes mass, momentum, and energy exchange between the surface and subsurface flows. A few experimental and numerical studies have provided some evidence that permeability can indeed influence the properties of the surface flow. Zagni and Smith ${ }^{1}$ conducted experiments with open channel flows over permeable beds composed of spheres. The size and grading of the bed material were varied extensively in order to investigate the effects of various beds having different values of permeability on the friction coefficients. The main finding was that even at Reynolds numbers for which one would expect hydraulically rough flow-regime friction factors for permeable beds in- 


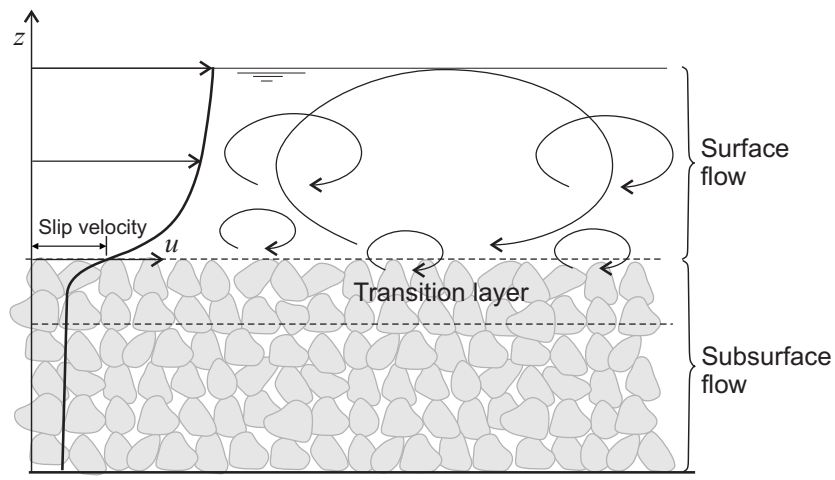

FIG. 1. Flow subdivision in open channel flows over permeable beds, the solid line represents a possible mean velocity profile.

creased with increasing Reynolds number. Zippe and $\mathrm{Graf}^{2}$ performed experiments comparing measurements taken over permeable and impermeable beds having the same roughness texture in order to isolate the effects of permeability on the surface flow. The bed material was composed of spheres and velocities were measured by means of a single velocity component hot film anemometer. The flows investigated were in the fully rough regime since the size of the roughness elements was much larger than the viscous length scale. Results showed that the friction factor was higher for the permeable case and it was Reynolds number dependent, hence substantiating the findings of Zagni and Smith. ${ }^{1}$ Furthermore Zippe and $\mathrm{Graf}^{2}$ observed that the normalized intensity of the streamwise velocity fluctuations for the permeable and impermeable wall collapsed reasonably well.

Recently, Breugem et al. ${ }^{3}$ presented Direct Numerical Simulations (DNS) of the volume-averaged Navier-Stokes equations for a channel flow bounded by a smooth wall at the top and a permeable wall at the bottom. The volume averaging procedure was applied to a flow region big enough to smooth inhomogeneities at the pore scale but also small enough to resolve the flow dynamics of interest at larger scales. This approach, besides being computationally less demanding than a standard DNS proved to be reliable for the description of flow statistics above and within the permeable wall. Breugem et al. ${ }^{3}$ point out that permeable walls are also unavoidably rough. The effect of surface roughness on the turbulent flow depends on the roughness Reynolds number defined as $\operatorname{Re}_{d}=d u_{*} / \nu$, where $d$ is the characteristic diameter of the particle composing the bed. The effects of permeability on the flow may be quantified by the permeability Reynolds number defined as $\operatorname{Re}_{K}=\sqrt{K} u_{*} / \nu$, where $K$ is the wall permeability and $\sqrt{K}$ is a length scale of turbulence acting at the pore scale. $\mathrm{Re}_{K}$ represents a ratio between effective pore diameter and the viscous length scale $\nu / u_{*}$. For large values of $\mathrm{Re}_{K}$ eddies are able to penetrate the permeable wall and the effects of permeability are significant, whereas for $\operatorname{Re}_{K}$ $\ll 1$ the wall can be considered impermeable. Breugem et al. ${ }^{3}$ aimed at studying the effects of permeability exclusively; therefore a small particle diameter was imposed in the simulations in order to have a small $\operatorname{Re}_{d}$ and high $\operatorname{Re}_{K}$. This guaranteed that the effects of permeability were enhanced and those of roughness minimized. The analysis of mean velocity profiles showed that the higher the bed permeability, the higher the resistance imposed on the flow. When compared to the smooth wall case, vertical profiles of turbulence intensity (i.e., rms of velocity fluctuations) for the permeable wall showed a decrease in the peak of the streamwise velocity component and an increase in the peak of the spanwise and wall-normal components. This effect was explained as a consequence of the absence of longitudinal low- and highspeed streaks destroyed by the turbulent transport across the permeable surface.

The main outcome from the studies listed above is that friction factors for permeable beds are Reynolds number dependent even in the hydraulically rough regime and, in general, are higher than the friction factors related to impermeable beds having identical roughness texture. Zagni and Smith ${ }^{1}$ claimed that the flow mechanism responsible for such an increase in friction factors is associated to shear penetration within the permeable bed. In other words, they argued that the increase in flow resistance is associated to a more efficient energy dissipation caused by the exchange of momentum between the surface and subsurface flows, across the bed surface. The main shortcoming of the previous experimental studies is that they are mainly focused on first order velocity statistics and the effects of permeability on other relevant turbulence quantities have been largely neglected.

\section{B. Turbulence properties of the transition layer}

In existing literature, there are many experimental and numerical studies of the flow properties of the transition layer in the case of laminar boundary layers. ${ }^{4-12}$ In contrast, due to technical difficulties and heavy computational costs, the number of experiments and numerical simulations explicitly designed for investigating the transition layer between a turbulent boundary layer and a porous medium region is much more limited. ${ }^{13-15,3}$

Ruff and Gelhar ${ }^{13}$ performed velocity measurements in pipe flows where the porous medium was composed of foam. Velocities were measured by means of hot-wire anemometry. Results showed that mean velocities and turbulence intensities decayed exponentially within the porous medium.

Another experimental attempt to gain insight into the turbulence characteristics of the subsurface flow was presented by Vollmer et al., ${ }^{15}$ where pressure fluctuations were measured at different levels within a gravel bed of an open channel flow, in order to investigate the effects of coherent structures on the exchange of solutes between the surface and subsurface flows. The rms of pressure fluctuations were found to decrease exponentially inside the bed where the high frequency components of the pressure signal vanished more rapidly than low frequency components. It was also argued that high frequency pressure fluctuations were caused by "bursting" events such as sweeps and ejections, whereas low frequencies were induced by the large scale flow structures scaling with the water depth.

Although the main focus of the numerical work by Breugem et $\mathrm{al}^{3}{ }^{3}$ was investigating the effects of permeability on the surface flow, plenty of results are also presented about the flow within the permeable wall. The simulations of Breu- 
gem et $a l^{3}$ confirmed the exponential decay of turbulence intensities, pressure fluctuations, and mean velocities within the permeable bed.

It is clear that the properties of turbulence within the transition layer have been poorly investigated. In particular, experimental studies on this topic are scarce (only the studies from Ruff and Gelhar ${ }^{13}$ and Vollmer et al. ${ }^{15}$ provided some insight). More needs to be done to provide data for future model validations and also to gain more insight into the transport mechanisms responsible for the coupling between the surface and the subsurface flow.

\section{Aims and objectives}

Within the context of the study of open channel flows over permeable beds, the general objectives of the present paper are as follows: (i) investigating the influence of permeability on the turbulence properties of the surface flow, with particular focus on flow resistance, second order velocity statistics, and the vertical distribution of shear stress components; (ii) investigating, with unprecedented experimental detail, the turbulence properties of the subsurface flow within a packed bed underlying turbulent open channel flows at two different Reynolds numbers.

In order to fulfill the objectives listed above, we have designed and carried out two sets of experiments: one set involved open channel flows over a single layer of spheres, while for another one the bed was made of five such layers. The one layer bed was considered as rough and impermeable, whereas the five layer bed was considered as rough and permeable. The main difference between the two is that the latter allows for the surface flow to interact with a volume of fluid below the bed surface, which is much larger than the typical size of the bed roughness. Since the two bed configurations are characterized by the same roughness texture, the comparison of surface flow velocity statistics between the two allowed to isolate the effects of permeability.

The permeable bed chosen for the experiments is characterized by a very large value of $\operatorname{Re}_{K}$ (Table I), which according to Breugem et al., ${ }^{3}$ classifies the bed as highly permeable. It is therefore plausible to expect differences in surface flow characteristics between the permeable and impermeable bed cases.

The experimental data pertaining to the surface flow velocity statistics were computed and interpreted following the double averaging methodology (DAM). More details on the characteristics of such methodology and on its application to our set of data are provided later in the text.

The packing of the spheres composing the bed was chosen in order to allow velocity measurements to be performed within the voids of the permeable bed and therefore to investigate the turbulence properties of the subsurface flow. Such measurements helped identifying the dominant scales of turbulence characterizing the subsurface flow and to clarify the transport mechanisms which control the exchange of turbulent kinetic energy (TKE) between the surface and subsurface flows.

Following the description of the experimental procedures and methods presented in Sec. II, this paper is orga-
TABLE I. Hydraulic conditions for the experiments; $H$ is the flow depth measured from the bead tops to free surface; $\mathrm{S}$ is the slope of the bed; Re is the bulk Reynolds number; $\operatorname{Re}_{K}$ is the permeability Reynolds number; $U_{b}$ is the depth averaged velocity; $R$ is the hydraulic radius; $K$ is the permeability of the bed; $\phi$ is the porosity of the bed; $\tau_{r}$ and $\tau_{H}$ are the bed shear stress calculated as in Eqs. (1) and (2), respectively; $\tau_{R S}$ is the bed shear stress obtained from extrapolation of the spatially averaged Reynolds stress profile to the bead tops; $f=u_{*}^{2} / U_{b}^{2}$ is the friction factor.

\begin{tabular}{|c|c|c|c|c|}
\hline & \multicolumn{2}{|c|}{ Five layers } & \multicolumn{2}{|c|}{ One layer } \\
\hline & Low $\mathrm{Re}$ & High Re & Low Re & High Re \\
\hline$H(\mathrm{~mm})$ & 20 & 42 & 22 & 42 \\
\hline $\mathrm{S}(\%)$ & 0.25 & 0.25 & 0.25 & 0.25 \\
\hline$U_{b}(\mathrm{~m} / \mathrm{s})$ & 0.254 & 0.39 & 0.28 & 0.42 \\
\hline $\operatorname{Re}=U_{b} H / \nu$ & 5588 & 16380 & 6160 & 17640 \\
\hline$\tau_{R}\left(\mathrm{~N} / \mathrm{m}^{2}\right)$ & 0.445 & 0.852 & 0.484 & 0.852 \\
\hline$\tau_{R S}\left(\mathrm{~N} / \mathrm{m}^{2}\right)$ & 0.462 & 0.948 & 0.529 & 0.906 \\
\hline$\tau_{H}\left(\mathrm{~N} / \mathrm{m}^{2}\right)$ & 0.488 & 1.030 & 0.538 & 1.030 \\
\hline$u_{*}=\sqrt{\tau_{R S} / \rho}(\mathrm{m} / \mathrm{s})$ & 0.0215 & 0.0308 & 0.0230 & 0.0301 \\
\hline $\operatorname{Re}_{K}=\sqrt{K} u_{*} / \nu$ & 31.2 & 44.6 & $\ldots$ & $\ldots$ \\
\hline$f=u_{*}^{2} / U_{b}^{2}$ & $7.17 \times 10^{-3}$ & $6.23 \times 10^{-3}$ & $6.75 \times 10^{-3}$ & $5.14 \times 10^{-3}$ \\
\hline$K\left(\mathrm{~mm}^{2}\right)$ & & 2.08 & & \\
\hline$\phi(\%)$ & & $48 \%$ & & \\
\hline
\end{tabular}

nized as follows: Sec. III is devoted to the description and interpretation of the results. This section is divided into two parts: The first deals with the effects of permeability on the surface flow whereas the second includes the results obtained from the subsurface flow measurements. Then, in Sec. IV we present a general discussion of the experimental results and Sec. V summarizes all the main findings of the paper.

\section{EXPERIMENTAL TECHNIQUE AND PROCEDURES}

All the experiments were carried out using an $11 \mathrm{~m}$ long tilting hydraulic flume with a $0.4 \mathrm{~m}$ wide rectangular cross section. The bed was covered with $12 \mathrm{~mm}$ diameter glass balls packed in a cubic pattern and arranged in two bed configurations: one characterized by one layer of spheres (considered as the impermeable bed) and another by five layers (considered as the permeable bed). The cubic-packed bed extended in both cases for half of the flume length, i.e., for $5.5 \mathrm{~m}$. All experiments were conducted at uniform fully developed turbulent rough flow conditions. The width-to-depth ratio was kept in all cases higher than 5, for which it is possible to assume that the flow was two dimensional, at least in the central part of the flume. ${ }^{16}$ The experiments were conducted with two Reynolds numbers for each bed configuration. Hereafter we refer to these two flow conditions as the low and high Reynolds number (Re) experiments (Table I). The permeability of the five layer bed reported in Table I was determined using a separate rig similar to conventional "Darcy apparatus." Please see Ref. 17 for a detailed description of the experimental apparatus and the analysis of the data. 


\section{A. Surface flow measurements}

For both bed configurations velocity measurements within the surface flow were carried out by means of particle image velocimetry (PIV). PIV is a quantitative flow visualization technique that relies on the illumination of a plane area of the flow, which is seeded with small neutrally buoyant particles. In these experiments, hollow glass spheres with a mean diameter of $15 \mu \mathrm{m}$ were used as the seeding material. PIV images were obtained from vertical planes of the flume by repeatedly illuminating the flow measurement area with a double pulsed Nd-YAG (yttrium aluminum garnet) laser (frequency doubled to produce visible green light), which was synchronized with a digital camera [Kodak (roper) megaplus ESI] that records image pairs with a 1008 $\times 1008$ pixels resolution, directly to a high-specification PC with frame-grabbing hardware. VIDPIV analysis software was then used to analyze the images in order to work out the velocity field. The software applies a cross-correlation algorithm, for each image pair, to regions of the flow called interrogation areas, where it determines the average particle displacements in pixel units. Hence, with the knowledge of the time delay between laser pulses and the scale of the digital image, the software calculates particle (and flow) velocities. Interrogation areas can be overlapped to increase the number of measurement points and better resolve spatial characteristics of the flow.

In our experiments velocities were measured along the bed-parallel and bed-normal directions, which are here denoted with $(u)$ and $(w)$, respectively. The laser light sheet was placed at two streamwise oriented vertical planes (Fig. 2): one over the tops of the balls (top section) and another over their connections (valley section). The spanwise position of the valley and top sections was chosen as close as possible to the central cross section of the flume. For each measurement section, the flow region investigated covered at least one sphere diameter in length. This allowed to have enough measurement detail to estimate spatially averaged flow statistics. ${ }^{18}$

For each experiment, 2200 images were recorded at a sampling frequency of roughly $13 \mathrm{~Hz}$. The random error of a PIV measurement was estimated to be $\Delta v=0.0025 \mathrm{~m} / \mathrm{s}$. It was computed as $\Delta v=\Delta s / \Delta t$, where $\Delta s$ corresponds to $1 / 10$ of 1 pixel physical dimension, and $\Delta t$ is the time delay between the subsequent images. ${ }^{19}$ For each experiment the time delay was set as $\Delta t=0.001$ s. PIV measurements were validated by checking the closure for momentum balance in the surface flow. This was carried out by comparing the shear stress obtained from the linear interpolation of spatially averaged Reynolds stress to the level of the bead tops and the shear stress estimated from global momentum balance; the latter was calculated in the following two ways:

$$
\begin{gathered}
\tau_{R}=\rho g S R, \\
\tau_{H}=\rho g S H,
\end{gathered}
$$

where $\rho$ is the water density, $g$ is the acceleration due to gravity, $S$ is the slope of the bed, $R$ is the hydraulic radius, and $H$ is the flow depth measured from the bead tops to the

\section{Side view}

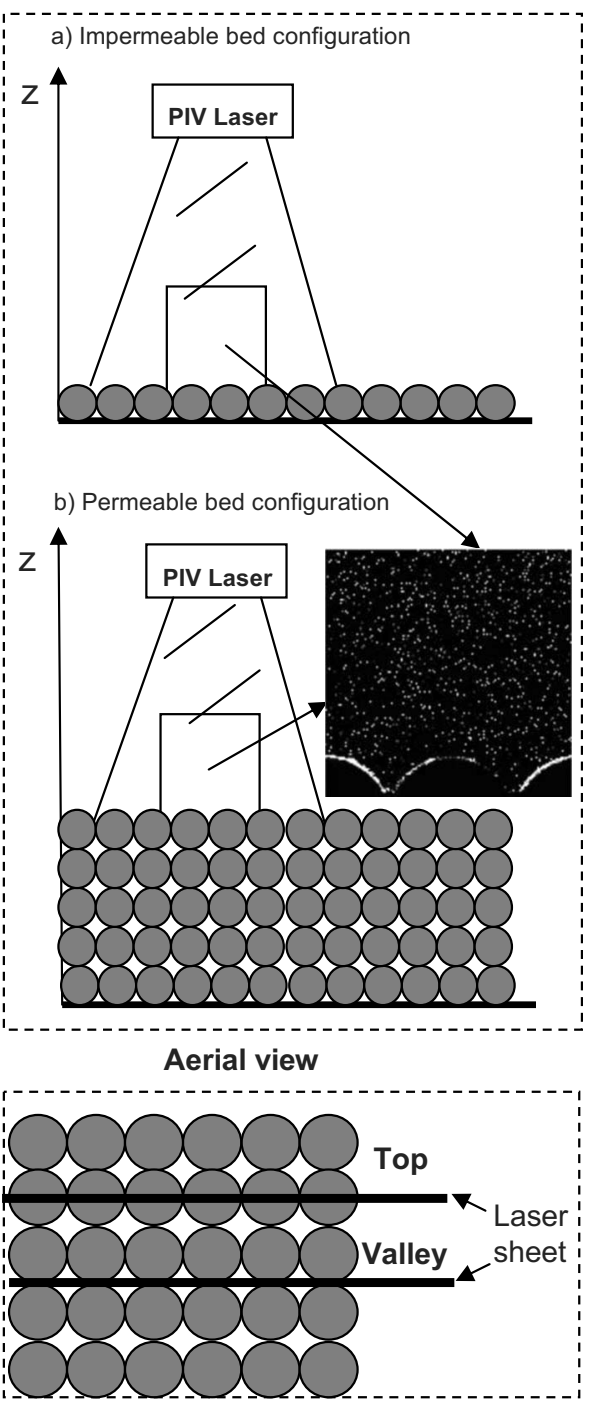

FIG. 2. Measurement window for the PIV experiments and positioning of the laser sheet.

water free surface. The representative value of the shear stress at the measurement location should lie between the estimates provided by Eqs. (1) and (2), respectively. ${ }^{20}$ Equation (1) provides the minimum possible value for the shear stress since it is estimated assuming that the flow resistance exerted by the sidewalls of the flume is the same as that exerted by the bed. Instead Eq. (2) provides the maximum value since the sidewall effects are neglected. It is encouraging to see that the shear stress estimated from the Reynolds stress profiles lies between $\tau_{R}$ and $\tau_{H}$ for all the experiments (Table I).

\section{B. Subsurface flow measurements}

Velocities within the porous medium were measured by means of a Met-Flow, ultrasonic velocity profiler (UVP). ${ }^{21}$ UVP consists of a cylindrical probe $(60 \mathrm{~mm}$ long and $8 \mathrm{~mm}$ in diameter) generating a short emission of ultrasound (US), which travels along the measurement axis, and then hits particles in the water, which reflect part of the US energy. The "echo" generated by the particles is then detected by the 
(a)
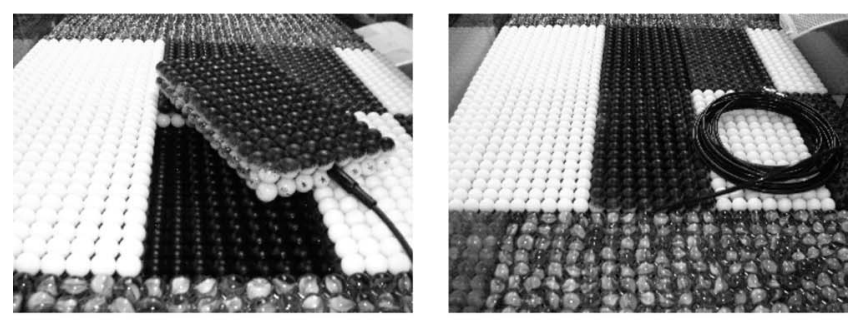

(b)

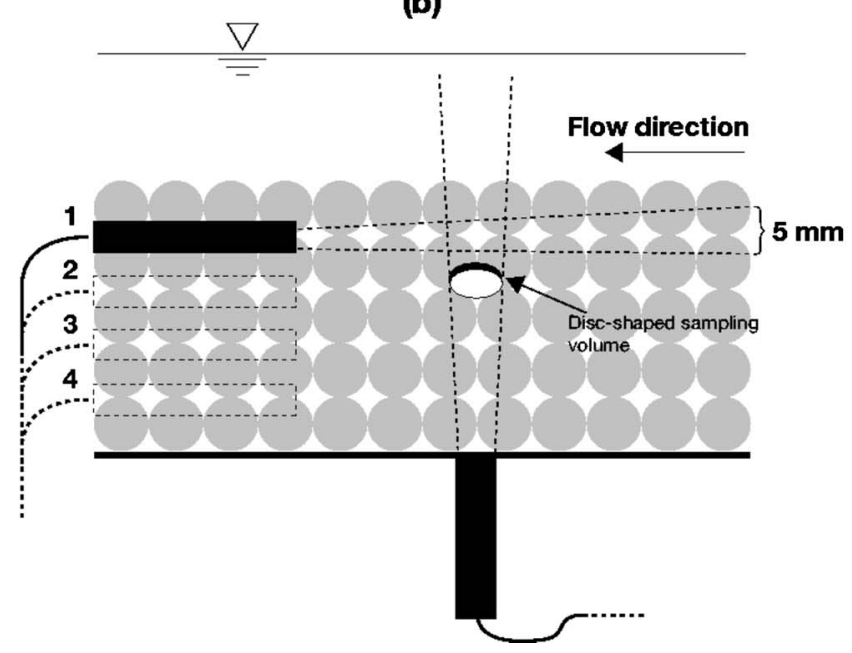

FIG. 3. Display of the UVP probe within the porous medium; (a) UVP probe inserted into the top pore; (b) positions of the UVP probe within the bed, i.e., pores $1-4$.

probe which determines the shift in frequency and the associated particle velocity component along the US propagation axis. UVP can measure instantaneous velocities at many locations along the measurement axis, permitting calculation of both time and space-averaged flow variables. In this application, velocities were measured by means of a $4 \mathrm{MHz}$ Met-Flow UVP probe, ${ }^{21}$ which was inserted in a hole drilled in the bed and oriented along the flow, as shown in Fig. 3(a). The probe measures instantaneous velocities at 128 locations or bins within a length of about $95 \mathrm{~mm}$. Each measurement bin is characterized by a disk shaped sampling volume with a $0.74 \mathrm{~mm}$ thickness and an $\sim 5 \mathrm{~mm}$ diameter. The bin diameter grows with distance from the probe head [Fig. 3(b)] due to the divergence of the US beam. Each bin is independent from the other and there is no overlapping among them.

The echo signal detected by the probe was monitored with an oscilloscope, which was particularly helpful for recognizing and then removing obstacles accidentally standing along the measurement axis such as air bubbles or debris particles. In the location of the UVP measurements, glass beads were replaced with a $40 \mathrm{~cm}$ strip of plastic beads (Delrin plastic material) with the same diameter $(12 \mathrm{~mm})$ and the same cubic packing. Plastic beads were used because variation in their diameters was appreciably lower compared to the glass beads, and this helped avoiding anomalous reflections of U.S. from the beads. Furthermore, it is much easier to drill holes through plastic than through glass. The holes were used for inserting the UVP probe within the pores of the bed. For the remaining part of the flume, glass beads were preferred because of their much lower cost.

In order to improve the quality of the echo signal, seeding was added upstream the test section; instant coffee proved to be excellent for this purpose. During the flume experiments the bed-parallel velocity component $u$ was measured at several heights of the bed moving the UVP probe at four positions (pores 1-4) whereas bed-normal velocities $w$ were measured placing the probe perpendicularly to the flume [Fig. 3(b)]. The use of only one UVP probe did not allow the simultaneous measurement of both velocity components.

In a UVP system, the velocity resolution $V_{\min }$ (i.e., the minimum measurable velocity) can be tuned by setting a maximum measurable velocity $V_{\max }$. The lower $V_{\max }$ the smaller $V_{\text {min }}$, i.e., the higher the resolution of velocity measurements. ${ }^{21}$ The former was chosen just big enough to cover the higher velocity magnitudes in order to have the lowest possible $V_{\min }$. The $V_{\min }$ for bed-parallel velocities was in the range of $0.8-1.1 \mathrm{~mm} / \mathrm{s}$, whereas for the vertical component $V_{\min }$ was $0.7-0.9 \mathrm{~mm} / \mathrm{s}$.

All the advantages and shortcomings of UVP for measuring velocities within packed beds are discussed in depth in Ref. 22. Nevertheless, here we list the main results from this previous study: (i) the UVP probe influences the pore flow only in the first $10 \mathrm{~mm}$ along the measurements axis; (ii) instantaneous UVP measurements at each location represent an average velocity of a fluid parcel contained within the disk shaped sampling volume characterizing each measurement point; (iii) despite the divergence of the US beam, flow measurements are reliable and reasonably uniform for at least a length of $90 \mathrm{~mm}$ from the probe head.

\section{RESULTS}

In the following text, all the results are presented by plotting velocity statistics using the roughness tops as the origin for the vertical coordinate. The shear velocity used for normalization of velocity statistics is defined as $u_{*}=\sqrt{\tau_{R S} / \rho}$.

The velocity statistics pertaining to the surface flow were analyzed and computed following the DAM. This methodology relies on an averaging procedure which follows two steps: (i) time averaging is applied in order to compute flow statistics for each measurement point contained within the flow regions captured by PIV; (ii) flow statistics resulting from time averaging are spatially averaged along thin boxes parallel to the bed. ${ }^{18}$ The streamwise extent of such boxes is one bead diameter and, in the lateral direction, they include measurement points located in the top and valley section. By virtue of the PIV methodology the thickness of the averaging boxes is equal to the height of the interrogation areas. Ideally, at each elevation, the averaging boxes should contain enough measurement points to cover all the spatial heterogeneity of the near-bed flow. In our experiments PIV measurements were performed at two lateral sections, where we expected to capture the largest spatial variations of the flow. Spatially averaged statistics computed from more detailed 
measurements along the lateral direction may slightly differ from those presented in this paper.

The two-step averaging procedure described above was applied to compute the following spatially averaged flow statistics: $\left.\left.\langle\bar{u}\rangle, \overline{\left\langle u^{\prime 2}\right.}\right\rangle, \overline{\left\langle w^{\prime 2}\right.}\right\rangle$, and $\left.\overline{\left\langle u^{\prime} w^{\prime}\right.}\right\rangle$, where the straight overbar symbol stands for time averaging, the prime index identifies time fluctuations (e.g., $u^{\prime}=u-\bar{u}$ ), and angular brackets denote spatial averaging. In order to gain insight into the effects of permeability on the flow structure characterizing the near-bed flow region, we also present some velocity statistics which describe the variability and coherence of the time-averaged flow. This includes the "intensity of the spatial variability" and covariance of time-averaged velocities, i.e., $\sqrt{\left\langle\widetilde{u}^{2}\right\rangle}, \sqrt{\left\langle\widetilde{w}^{2}\right\rangle}$, and $\langle\tilde{u} \widetilde{w}\rangle$, where the wavy overbar identifies the spatial variations of the time-averaged flow with respect to the double-averaged (DA) flow (i.e., $\widetilde{u}=\bar{u}$ $-\langle\bar{u}\rangle)$. The two quantities $\sqrt{\left\langle\tilde{u}^{2}\right\rangle}$ and $\sqrt{\left\langle\widetilde{w}^{2}\right\rangle}$ and the covariance $\langle\tilde{u} \widetilde{w}\rangle$ are usually called normal and shear "forminduced" stress, respectively. ${ }^{23,24}$ The latter represents a fluid shear stress term appearing within the DA momentum equations. In particular, $\langle\tilde{u} \widetilde{w}\rangle$ accounts for the momentum flux induced by the heterogeneity and covariance of the bedparallel and bed-normal time-averaged velocities. ${ }^{25,26}$ For a complete review of the DAM methodology and the associated DA transport equations, see Refs. 27 and 28.

\section{A. Surface flow: A comparative analysis}

Figure 4 shows DA velocity and spatially averaged Reynolds stress for the permeable and impermeable bed experiments. Throughout the whole flow depth, DA velocities normalized with the shear velocity are lower above the permeable bed than those above the impermeable bed. The associated increase in friction factor is $21 \%$ in the high $\mathrm{Re}$ experiment and 6\% in the low Re experiment (Table I). The enhancement of friction factors due to permeability and their dependence on the Reynolds number is in line with the findings of Zagni and Smith ${ }^{1}$ and Zippe and Graf. ${ }^{2}$

In general, the vertical profiles of the spatially averaged turbulence intensities $\left(\sigma_{u} / u_{*}, \sigma_{w} / u_{*}\right)$ and shear Reynolds stress $\left(\left\langle\overline{u^{\prime} w^{\prime}}\right\rangle / u_{*}^{2}\right)$ follow curves which agree well with those typical of rough-bed open channel flows. In proximity of the bed surface, $\sigma_{u} / u_{*}$ and $\sigma_{w} / u_{*}$ reach the standard maxima of roughly 2 and 1 , respectively. Further above, $\sigma_{u} / u_{*}$ and $\sigma_{w} / u_{*}$ decrease with height following a well known exponential-law behavior. ${ }^{29,16}$ The spatially averaged shear Reynolds stress linearly increases with decreasing elevation until it reaches a maximum at $z / d=0.05$ and then decreases toward the bed surface. Such a decrease in $\left\langle\overline{u^{\prime} w^{\prime}}\right\rangle / u_{*}^{2}$ is due to non-negligible form-induced and viscous shear stress, contributing to momentum balance in the near-bed flow region (see Fig. 6 below and Ref. 18). Below the roughness crests, form and viscous drag are also responsible for the rapid decrease in the spatially averaged Reynolds stress. Unlike mean velocities, $\sigma_{u} / u_{*}, \sigma_{w} / u_{*}$, and $\left\langle\overline{u^{\prime} w^{\prime}}\right\rangle / u_{*}^{2}$ show no obvious difference between permeable and impermeable bed experiments, except for $\sigma_{u} / u_{*}$, which seem to be slightly smaller for the permeable bed.

The normalized TKE production term due to the work of
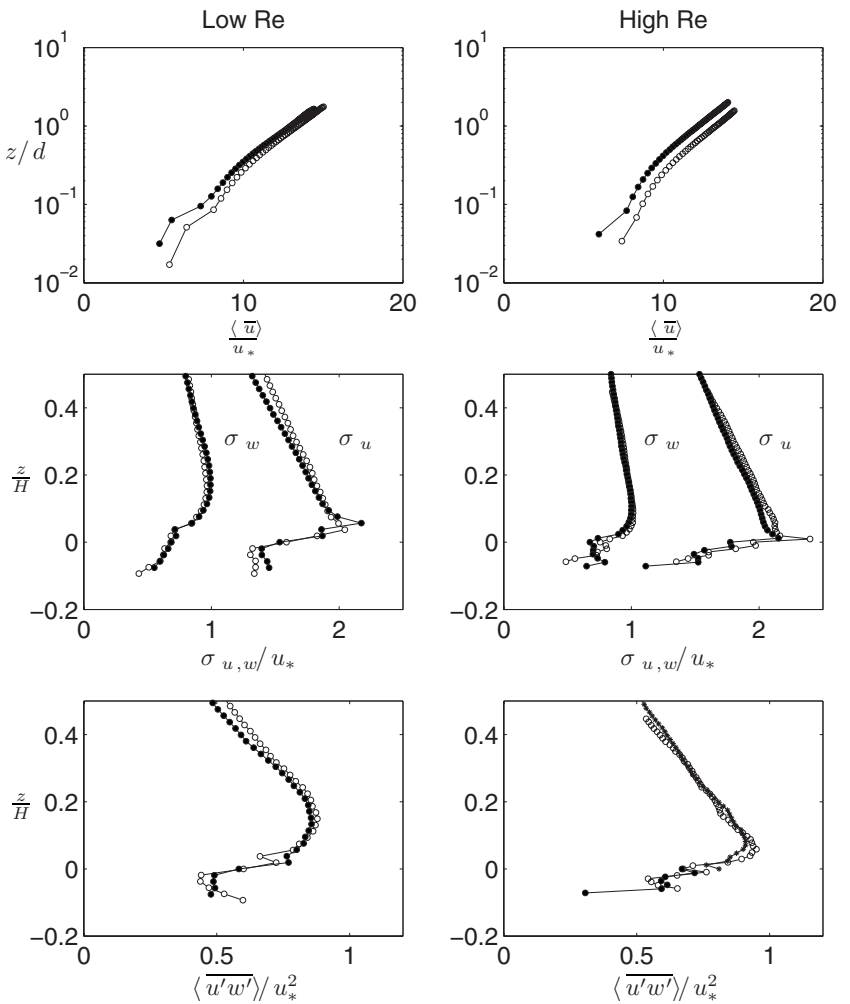

FIG. 4. Spatially averaged flow statistics. Open and filled symbols stand for one and five layer beds, respectively; $\sigma_{u}=\sqrt{\left\langle\overline{\left.u^{\prime 2}\right\rangle}\right.} ; \sigma_{w}=\sqrt{\left\langle\overline{w^{\prime 2}}\right\rangle} ; H$ is the flow depth; $u_{*}$ is the shear velocity as defined in Table I.

the spatially averaged Reynolds stress against DA mean flow (Fig. 5) is very similar for the permeable and impermeable beds at both Re. This means that the increase in the friction factor for the permeable bed is not a result of enhanced turbulence production within the surface flow. Therefore, as argued by Zagni and Smith ${ }^{1}$ such additional frictional drag must occur within the bed and probably as a result of enhanced shear penetration.

Figures 6 and 7 show $\sqrt{\left\langle\tilde{u}^{2}\right\rangle}, \sqrt{\left\langle\widetilde{w}^{2}\right\rangle}$, and $\langle\widetilde{u} \widetilde{w}\rangle$ normalized with the friction velocity $u_{*}$ and the local DA velocity $\langle\bar{u}\rangle$, respectively. As one would expect, these quantities are
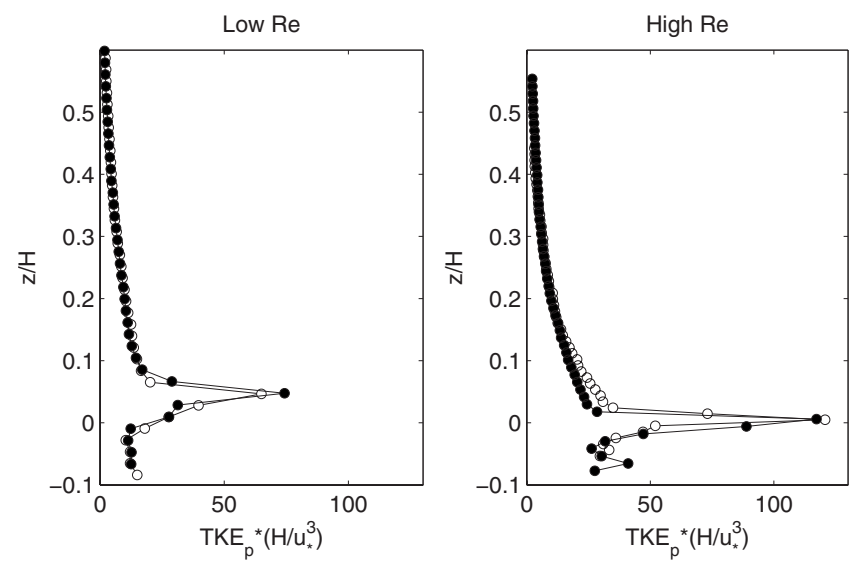

FIG. 5. TKE production term scaled with $u_{*}$ and flow depth $H$; TKE $p$ $=-\left\langle u \prime w^{\prime}\right\rangle(d\langle u\rangle / d z)$; open and filled symbols stand for one and five layer beds, respectively; $u_{*}$ is the shear velocity as defined in Table I. 

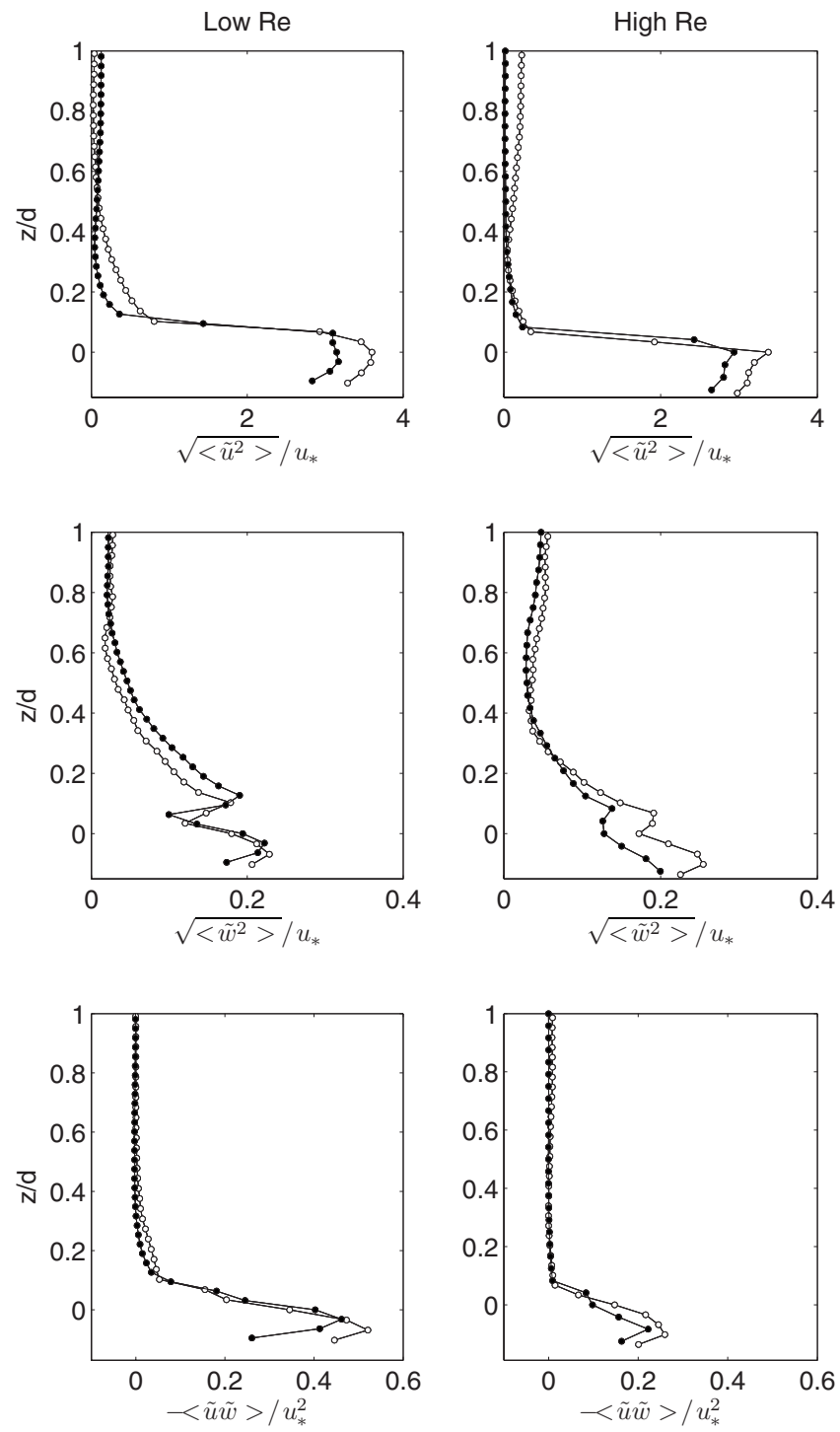

FIG. 6. Normal and shear form-induced stress normalized with $u_{*}$; open and filled symbols stand for one and five layer beds, respectively; $u_{*}$ is the shear velocity as defined in Table I; $d$ is the spheres' diameter.

higher in the near-bed region (where the time-averaged flow is directly influenced by the roughness topography) and then decrease with increasing height. $\sqrt{\left\langle\widetilde{u}^{2}\right\rangle}$ and $\sqrt{\left\langle\widetilde{w}^{2}\right\rangle}$ become negligible at $z / d>0.5$, whereas $\langle\widetilde{u} \widetilde{w}\rangle$ is already very small for $z / d>0.1$.

Interestingly, $\sqrt{\left\langle\tilde{u}^{2}\right\rangle} / u_{*}$ are higher for the impermeable bed experiments at both Re, whereas $\sqrt{\left\langle\widetilde{w}^{2}\right\rangle} / u_{*}$ show a Re dependence. Indeed, $\sqrt{\left\langle\widetilde{w}^{2}\right\rangle} / u_{*}$ is higher for the impermeable bed, at the high Re experiment, but lower at the low Re experiment. Consistently with $\sqrt{\left\langle\widetilde{u}^{2}\right\rangle} / u_{*}$, form-induced shear stress normalized with $u_{*}$ is slightly higher for the impermeable bed compared to the permeable bed

Overall, $\langle\bar{u}\rangle$ provides a much more effective velocity scale for the normalization of $\sqrt{\left\langle\tilde{u}^{2}\right\rangle}$ and $\sqrt{\left\langle\tilde{w}^{2}\right\rangle}$ since it provides a much better collapse of the data (Fig. 7). From a physical point of view, this means that the higher the DA velocities, the stronger are the spatial variations of the timeaveraged flow.
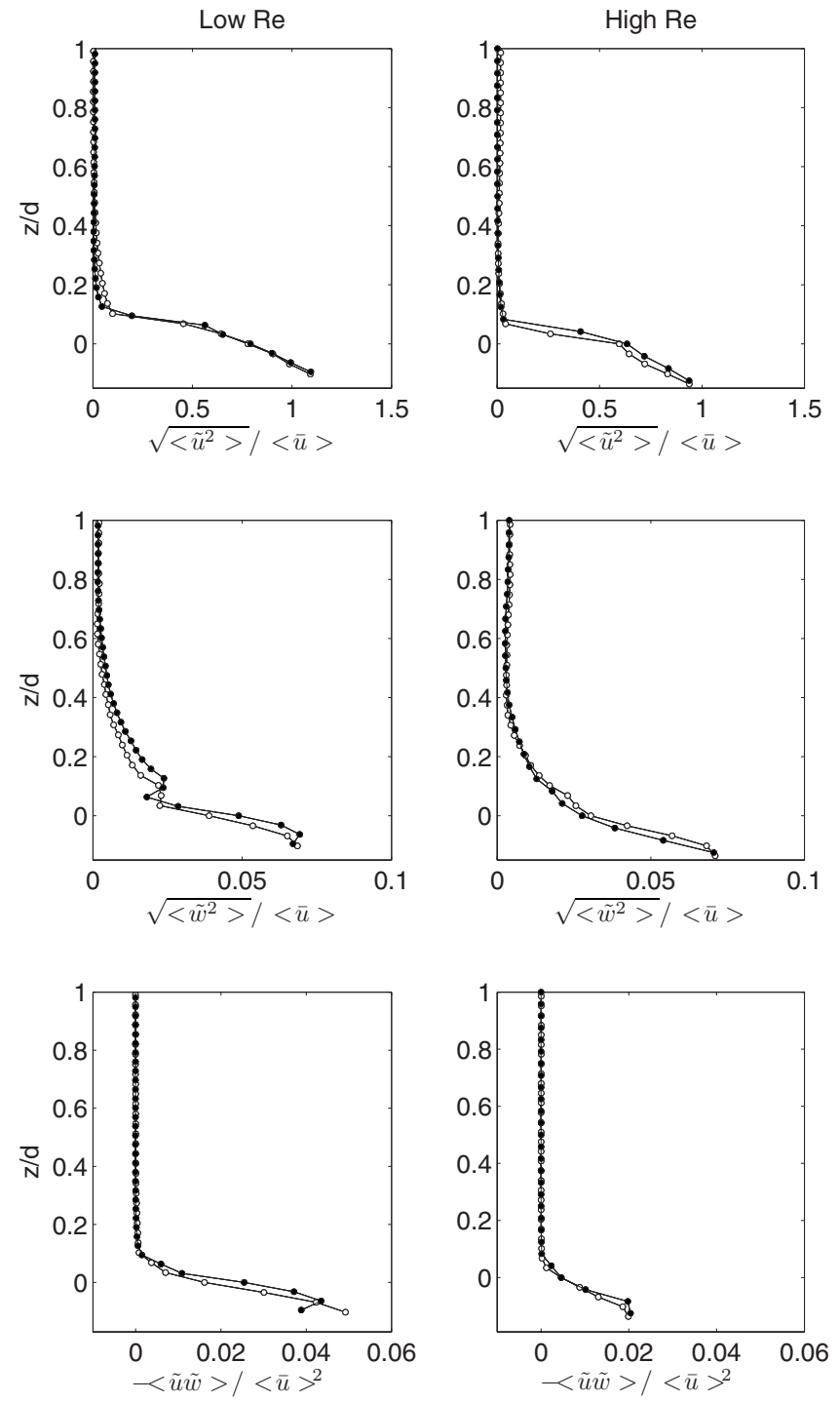

FIG. 7. Normal and shear form-induced stress normalized with $\langle\bar{u}\rangle$; open and filled symbols stand for one and five layer beds, respectively; $d$ is the spheres' diameter.

\section{B. Subsurface flow}

We now investigate the turbulence properties of the subsurface flow. The reader should be aware that in this section, we include figures where PIV and UVP data (pertaining to the surface and subsurface flows, respectively) are shown together in order to provide a complete picture of the whole flow domain, over and below the bed surface. However, the velocity statistics computed from the two sets of data are not perfectly comparable since PIV and UVP measure velocities within significantly different sampling volumes. PIV data are used to plot velocity statistics for $z / d>-0.17$, whereas UVP data for $z / d<0$. A small overlap of results obtained from the two measurement systems appear for the flow region -0.17 $<z / d<0$ in Figs. 10(b) and 12(b) below, which show the second and third order moments of the vertical velocity component.

By means of the horizontal deployment of the UVP probe within the packed bed, it was possible to measure bedparallel velocities at many points along the US propagation 
High Re

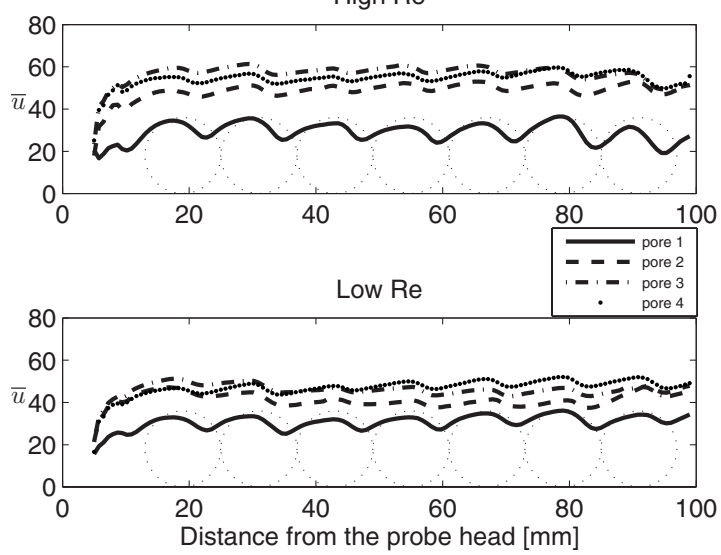

FIG. 8. Horizontal profiles of time-averaged velocities measured within the porous medium. Time-averaged velocities $\bar{u}$ are in $\mathrm{mm} / \mathrm{s}$.

axis and hence assess the spatial variability of the timeaveraged flow induced by the spheres. Figure 8 shows timeaveraged bed-parallel velocities $\bar{u}$ measured by the UVP at all four levels within the bed. Due to continuity, timeaveraged velocities increase in the narrower sections of the pores and decrease in the larger ones. This gives the wavy shape profile of $\bar{u}$ shown in Fig. 8.

In analogy with the surface flow, bed-parallel velocities measured by UVP, within the subsurface flow, were averaged first in time and then in space. However, for UVP, the spatial averaging window covers a flow region placed between 12 and $97 \mathrm{~mm}$ (seven bead diameters) from the face of the probe. The first $12 \mathrm{~mm}$ of the measurement window were neglected because the velocities in this region were influenced by the probe itself. ${ }^{22}$ DA velocities are shown together in Fig. 9 for all flow conditions.

In the upper parts of the bed, where the flow is strongly affected by the turbulent stream, DA velocity profiles show a peculiar feature: intuitively one would say that mean velocities within the subsurface flow should decrease uniformly within the bed due to the weakening of the shearing effect induced by the surface flow. In the experiments presented herein, this happens for the flow region $0<z / d<-0.17$ (a)

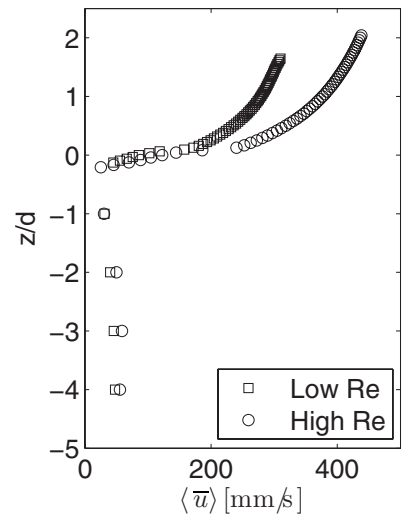

(b)

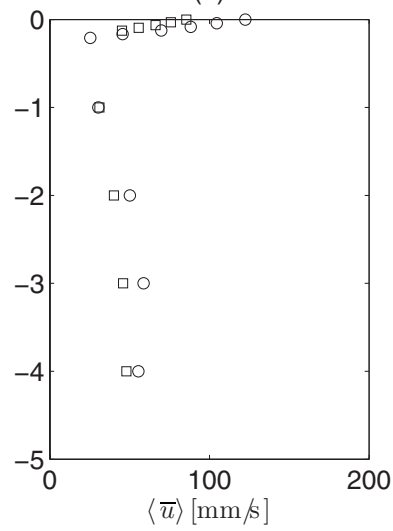

FIG. 9. DA velocity profiles for (a) the whole flow field, (b) the subsurface flow; $d$ is the spheres' diameter. (a)

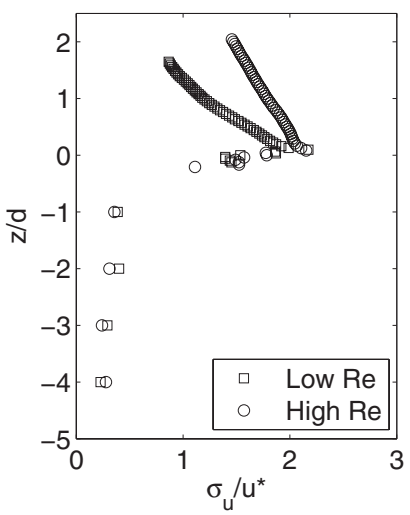

(b)

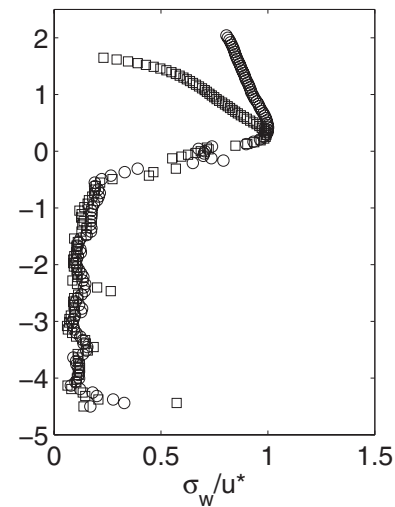

FIG. 10. Turbulence intensity profiles; $\sigma_{u}=\left\langle\sqrt{\overline{u^{\prime 2}}}\right\rangle ; \sigma_{w}=\left\langle\sqrt{\overline{w^{\prime 2}}}\right\rangle$ for $z / d>0$; $\sigma_{w}=\sqrt{\overline{w^{\prime 2}}}$ for $z / d<0 ; u_{*}$ is the shear velocity as defined in Table I; $d$ is the spheres' diameter.

where velocities decrease almost linearly with $z$ (measurements of linear velocity profiles within the roughness layer of various rough beds are also reported by Nikora et $\left.a l . .^{30}\right)$. In contrast, mean velocities $\langle\bar{u}\rangle$ below pore $1(z / d<-1)$ start to increase creating an unexpected dip in the velocity profile. The validation of this result as well as a plausible explanation for this anomalous behavior are discussed in Ref. 25 .

Figure 10 shows turbulence intensities. It is worth noting that due to the technicality of the measurements, the $\sigma_{w}$ profiles contain somewhat inconsistent data. While for PIV data it was possible to compute DA of the bed-normal velocity statistics; for the UVP data such spatial averaging could not be applied because velocities were measured only along a single vertical. The same applies to all the statistics involving the vertical velocity component.

Within the bed, turbulence intensity decays following an exponential form as observed by Ruff and Ghelar ${ }^{13}$ and Breugem et al. ${ }^{3}$ The exponential shape is well observable only from the profile of the bed-normal velocity component which, along the vertical, is resolved by many more points than the bed-parallel component.

Figure 10 shows that going from pore 4 to 1 (i.e., from $z / d=-4$ to $z / d=-1$ ), turbulence intensities $\sigma_{u} / u_{*}$ increase very slowly. This result may give the wrong perspective of the subsurface flow being characterized by a fairly uniform level of turbulence. However, such a slow increase can be justified by the significant drop of DA velocities observed in Fig. 9. In fact, turbulence intensities normalized with local DA velocities (Fig. 11) increase at a much higher rate, indicating that the turbulence activity significantly increases going from pore 4 to 1 .

Figure 12 shows the third order velocity moments for the bed-parallel and bed-normal velocity components, i.e., $M_{u u u}=\left\langle\overline{u^{\prime 3}}\right\rangle / \sigma_{u}^{3}$ and $M_{w w w}=\left\langle\overline{w^{\prime 3}}\right\rangle / \sigma_{w}^{3}$, respectively. $M_{w w w}$ gives indications on the rate and direction of TKE transport occurring by means of turbulent fluctuations. $M_{w w w}$ is positive above the roughness tops and negative below (for 0 $<z / d<-1.6)$ suggesting upward and downward TKE transports, respectively. For $z / d<-1.6, M_{w w w}$ oscillates around zero indicating that below a certain level, the transport of 


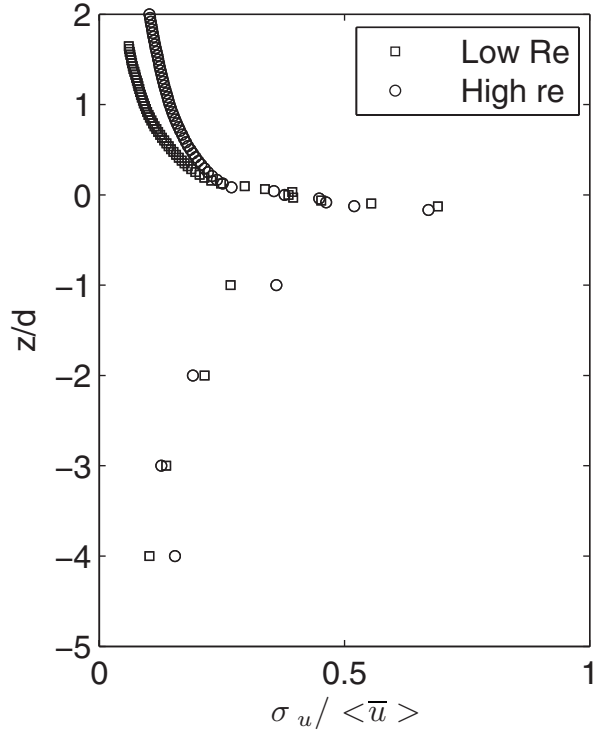

FIG. 11. Turbulence intensity of the bed-parallel velocity component normalized with local DA velocities; $\sigma_{u}=\left\langle\sqrt{\overline{u^{\prime 2}}}\right\rangle ; d$ is the spheres' diameter.

TKE (from the surface flow to the subsurface flow) by means of turbulent velocity fluctuations vanishes. $M_{\text {ии }}$ are negative throughout the whole subsurface flow, indicating that large values of bed-parallel velocity fluctuations occur mainly when they are negative.

\section{Two-point statistics and spectral analysis}

We now present two point statistics and spectral analysis to investigate the dominant scales of turbulence characterizing the subsurface flow. The analysis of two point statistics is here focused on the cross-correlation function of the type

$$
R H_{u u}(r, t)=\overline{u_{1}^{\prime}\left(x_{1}, t\right) u_{2}^{\prime}\left(x_{2}, t+\tau\right)} / \sigma_{u_{1}} \sigma_{u_{2}},
$$

where $r=\left|x_{2}-x_{1}\right|$ is the streamwise distance between two points taken along the measurement window, $\tau$ is the time lag, $u_{1}^{\prime}$ and $u_{2}^{\prime}$ are time fluctuations of the streamwise velocity component at positions 1 and 2 , respectively, and $\sigma_{1}$ and $\sigma_{2}$ represent the standard deviation of the streamwise velocity component at locations 1 and 2, respectively. Figure 13 (a)

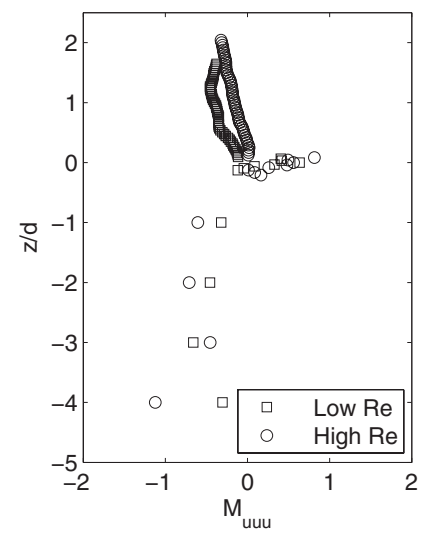

(b)

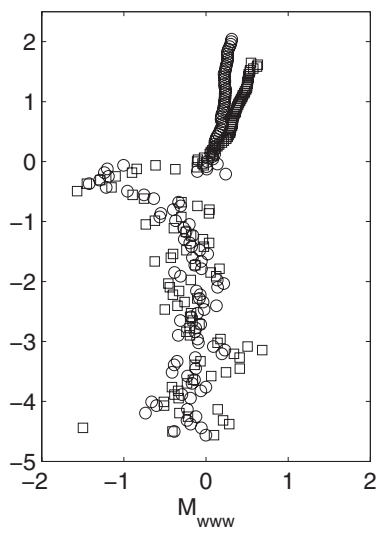

FIG. 12. Skewness profiles for (a) bed-parallel and (b) bed-normal velocity components; $M_{\text {uuu }}=\left\langle\overline{u^{\prime 3}}\right\rangle / \sigma_{u}^{3} ; M_{w w w}=\left\langle\overline{w^{\prime 3}}\right\rangle / \sigma_{w}^{3} ; d$ is the spheres' diameter.
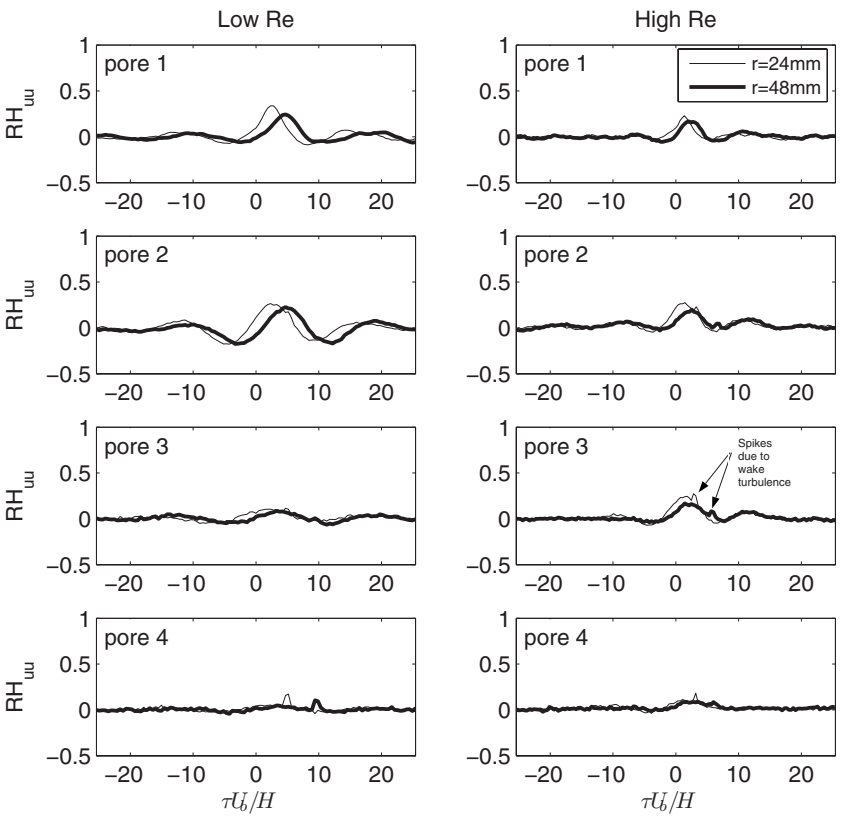

FIG. 13. Two point correlation functions; $U_{b}$ is the bulk mean velocity (Table I); $H$ is the flow depth.

shows cross-correlation functions calculated for spatial lags $r=24$ and $48 \mathrm{~mm} ; x_{1}$ was fixed at $24 \mathrm{~mm}$ from the head of the probe, in a position where the section of the pore is largest. Since $r$ was chosen as a multiple of the diameter of the spheres, (i.e., $d=12 \mathrm{~mm}$ ), $x_{2}$ was also positioned where the pore has its largest section.

At pores 2-4, the shape of $R H_{u u}$ indicates that the flow is characterized by eddies having two well distinguished turnover time scales $\Delta \tau$ : (i) a large one, which imposes nonnegligible values of $R H_{u u}$ for turnover time scales roughly ten times larger than $H / U_{b}$, which is the typical time scale of the largest eddies generated within the surface flow (i.e., $\Delta \tau \simeq 10 H / U_{b}$ ) and (ii) a small one which instead is responsible for the appearance of small spikes characterized by $\Delta \tau<H / U_{b}$. We argue that the large turnover time scales are caused by large scale turbulent motions generated in the surface flow and then imposed onto the subsurface flow. Instead, the small spikes are associated with pore-scale eddies locally generated within the porous medium in the form of wakes forming around the beads. It is not clear why secondary peaks do not appear at pore 1 . At this location the influence of the surface flow is strongest and turbulence intensities are significantly larger than in the lower pores (Figs. 10 and 11). It is plausible that the velocity fluctuations associated to such turbulent flow may be responsible to move wake eddies in and out of pore 1 . This means that such wake eddies may not be able to travel along pore 1 for distances equal to $r=\left|x_{2}-x_{1}\right|$ and therefore to contribute to $R H_{u u}(r, t)$.

Correlation maxima imposed by the large scale eddies decrease in magnitude from pore 1 to pore 4 , indicating that the energy of the large scales of motion imposed by the surface flow progressively weakens when they penetrate within the bed. Such weakening of large scale eddies is consistent with the decay of turbulence intensities observed in 


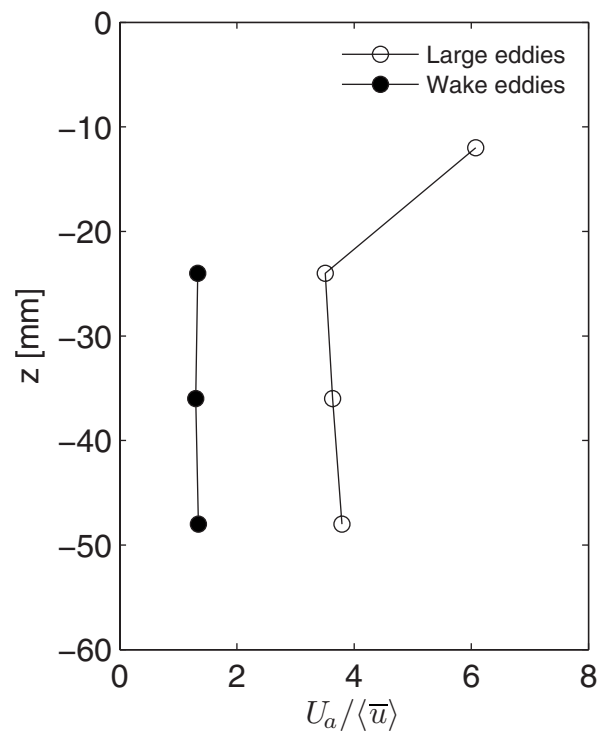

FIG. 14. Eddy convection velocity $U_{a}$ normalized with local mean velocity for large and small eddies within the porous medium. At pore 1 $(z=-12 \mathrm{~mm})$ the advection velocity for small scale turbulence could not be computed due to the absence of the secondary peaks in the cross-correlation function (Fig. 13).

Fig. 10. At pore $4, R H_{u u}$ display a weak contribution from large scale eddies so that pore-scale turbulence become the major contributors to correlation.

$R H_{u u}$ maxima for $r=24$ and $48 \mathrm{~mm}$, being associated to large or wake-scale eddies, are shifted along the $\tau U_{b} / H$ axis. This is a result of eddy advection. For one spatial lag $r$, the maxima of $R H_{u u}$ associated to large and wake eddies occur at different time lags, which suggest that these two scales of turbulence have a different advection velocity $U_{a}$. Such velocity can be estimated as the ratio between the spatial lag $r$ and the time lag at which the maxima in $R H_{u u}$ occur. Advection velocities for large and wake-scale eddies were calculated for the high Re experiments for which such maxima were most visible. $U_{a}$ for both eddy scales have been calculated for three values of $r$ in order to have a more robust statistics. Figure 14 shows that within the bed, large scale eddies move much faster than pore-scale eddies. Their advection velocity is indeed comparable with the bulk mean velocity $U_{b}$ of the surface flow. This confirms that such large scale eddies must be generated within the surface flow and then imposed within the subsurface flow. Instead, pore-scale eddies are generated locally because they move at velocities which are comparable with local/subsurface mean velocities $\langle\bar{u}\rangle(z)$.

Power spectra are usually obtained in the frequency domain as they are computed from single-point velocity measurements. It is possible to go from the frequency to the wave number domain by applying the Taylor hypothesis of frozen turbulence and hence have information on the spatial structure of turbulence. This hypothesis relies on the assumption that turbulent eddies at different length scales are advected by a velocity equal to the local mean velocity. In this study, the horizontal deployment of the UVP probe allowed having instantaneous measurements of $u$ at 128 points along the $x$ direction so that it was possible to compute spectra
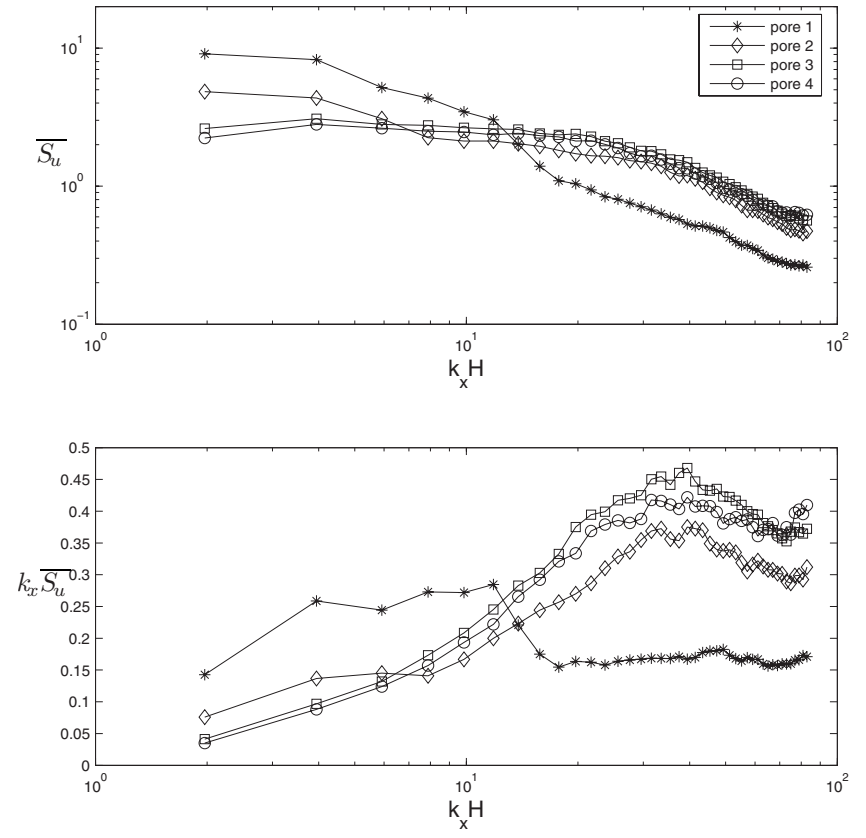

FIG. 15. Energy spectra in the wave number domain obtained from UVP measurements at pores 1-4 for low Re experiment; $H$ is the flow depth.

directly in the wave number domain $K_{x}=2 \pi / x$, where $x$ is the spatial scale of interest. This represents a great advantage since as we have observed from two point correlation functions, within the bed, eddies of different scales advect with different velocities and therefore the Taylor hypothesis is not applicable.

In order to obtain spectra directly in the wave number domain, one should consider measurements covering a length scale which contains the largest eddies in the flow so that each instantaneous measurement covers most of the turbulence scales of interest. This condition is best satisfied for the five layer low Re experiment, for which the measurement length covers up to four times the flow depth, which is roughly considered as the length of the largest eddies generated in turbulent open channel flows. ${ }^{31-33}$ We acknowledge that much larger scales have been recently observed in turbulent boundary layers and pipe flows (up to 20 times the boundary layer thickness, see Ref. 34) but their measurement and detection are well beyond the capabilities of our measurement system.

Spectra in the wave number domain were computed as follows: First, instantaneous spectra $S_{u}\left(k_{x}, t\right)$ were calculated for each instantaneous profile of velocity fluctuations $u^{\prime}(x, t)$ using a fast Fourier transform technique. Instantaneous spectra were also normalized with the variance of the velocities measured within each profile. Second, all the spectra obtained from instantaneous realizations of the flow were averaged at each wave number in order to decrease the confidence interval pertaining to each spectral estimate.

Spectra at all elevations are shown in Fig. 15. At pore 1, turbulent eddies at low wave numbers (within the range 2 $\leq k_{x} H \leq 10$ corresponding to eddies of size between one and four times the flow depth) are the major contributors to spectral energy (Fig. 15). However, moving from pore 1 to 4 , the contribution of low wave number components progressively 
decreases. The damping of spectral energy for such scales is consistent with the decrease in cross-correlation maxima observed in Fig. 13. Within the range of $2 \leq k_{x} H \leq 10$, such damping occurs faster for the higher than for the lower wave numbers. This is consistent with the concept of the porous medium acting as a low pass filter for surface flow turbulence. Such concept was first introduced in Ref. 15 where pressure fluctuations were measured within a gravel bed underneath an open channel flow. The rms of the pressure fluctuations were observed to quickly decrease within the bed and it was found that the bed dissipated high frequency fluctuations faster than the low frequency components; the signal of which was instead persistent even at the lowest bed levels.

At pores 2-4, power spectra develop a large peak at $k_{x} H \sim 40$ which corresponds to an eddy length scale of $\sim 3-4 \mathrm{~mm}$ [this is particularly visible in Fig. 15(b) where spectra are plotted in premultiplied form]. Such a spectral peak mirrors the appearance of the small spikes in the crosscorrelation functions $R H_{u u}$ of Fig. 13 and is associated to a wake type of turbulence generated by the flowing of water around the beads.

It should be noted that in general, in a fully developed turbulent flow $R H_{u u}$ computed with $r=24$ or $48 \mathrm{~mm}$ would not be able to capture the contribution of eddies with size of 3-4 $\mathrm{mm}$. This happens because eddies lose their coherency if they travel for distances much larger than their size. However, our experiments (at pores 2-4) were performed at a maximum pore Reynolds number $\operatorname{Re}_{p} \simeq 250$ which, according to Ref. 17, supports the occurrence of a transitional rather than a fully turbulent flow regime (using the same porous bed as in this paper, Horton and Pokrajac ${ }^{17}$ defined the pore Reynolds number as $\operatorname{Re}_{p}=\langle\bar{u}\rangle p / \nu$, where $p$ $=5 \mathrm{~mm}$ is the pore diameter). At this regime, wake eddies are occasionally shed from the spheres and are free to travel along the pores for fairly long distances without losing their coherency. Indeed, we observed (not shown here) that at pore 4 the secondary peak in $R H_{u u}$ is still visible up to lags $r=72 \mathrm{~mm}$.

\section{DISCUSSION}

In the present paper the effects of permeability on the subsurface flow have been isolated by comparing the velocity statistics of a flow over a single layer of spheres and a flow over five layers of spheres. The one layer case is considered as an impermeable bed which maintains the same surface roughness as the five layer bed. One may argue that a layer of half spheres could be a better choice than a layer of spheres because it is "more impermeable" while it still maintains the same surface roughness as the five layer bed. However, the use of spheres instead of half spheres allows evaluating the implications of some of our results for applications in river engineering. Indeed, hydraulic engineers usually estimate parameters related to flow resistance, such as the Manning coefficient, from the measurement of a characteristic diameter of the grains composing a river bed surface. By applying such a procedure, one would have predicted the same Manning coefficient for both our bed configurations. With our experiments we show that this is not correct be- cause the permeable bed has higher friction factors than the impermeable bed composed of identical grains. Therefore flow resistance of natural streams with highly permeable beds should be predicted by examining not just the layer of grains composing its surface but also the underlying porous structure. Using half spheres instead of spheres would mask this result because the equivalent diameter of half spheres is smaller than sphere diameter (it is between $d$ and $d / 2$ ) so it would have been perfectly plausible to observe higher friction factors for the permeable bed.

The experimental data presented in this paper allow identifying the mechanisms of production and transport of turbulence in open channel flows over permeable beds: the level slightly above the roughness tops represents the major source of TKE production (Fig. 5). Below this level, the turbulent transport of TKE diverges, splitting into upward and downward contributions (Fig. 12). The transport of TKE by means of turbulent velocity fluctuations vanishes one bead diameter below the roughness tops; however more TKEs are transported further down within the bed. Indeed cross-correlation functions and spectra (Figs. 13 and 15) clearly show how large scale eddies generated within the surface flow influence the subsurface flow even at the lowest bed levels. Since turbulent transport due to velocity fluctuations is not responsible for such TKE transfer, this must be promoted by pressure fluctuations. This finding is consistent with the results of Breugem et al.: ${ }^{3}$ in their simulations, the pressure transport term is the only one (among the other transport terms) that survives at the deepest positions within the permeable wall.

The analysis of our data revealed that in turbulent flows over permeable beds, there is a significant exchange of TKE between the surface and the subsurface flow. Therefore it appears surprising, to the authors, why such an interchange of energy does not impose any significant difference in the turbulence intensity profiles between the permeable and impermeable cases (Fig. 4). However, we also acknowledge that our results are limited to flows at relatively low Reynolds numbers. Since we have seen that the effects of permeability on the surface flow increase with increasing Re, it is plausible to expect more influence on the turbulence statistics as higher Re are approached.

\section{CONCLUSIONS}

Velocity measurements have been carried out in open channel flows over and within a permeable bed composed of five layers of beads. Comparison of flow statistics with an impermeable bed made of just one layer of such beads allowed investigating the influence of permeability on the surface flow statistics. Measurements of velocities within the five layer bed allowed investigating the turbulence properties of the subsurface flow and its interaction with the surface flow above. The analysis of results lead to the following main findings.

(i) The permeable bed offers a higher flow resistance than the impermeable bed. Flow resistance increases with increasing Reynolds number. As argued in Ref. 1 , such an increase seems to be caused by enhanced 
shear penetration within the bed whereas TKE production (scaled with the flow depth and the shear velocity) in the surface flow remains basically unaltered. (ii) Spatial variations of the time-averaged flow and the form-induced shear stress scale well with the local DA velocity. Since the permeable bed generates higher flow resistance than the impermeable bed, the same quantities, when scaled with $\mathrm{u}^{*}$, become larger for the impermeable bed.

(iii) Within the bed, we can recognize two types of turbulence, one generated at the pore scale and another one, more energetic, remotely generated within the surface flow and then imposed onto the flow within the bed. The associated transport of TKE by means of turbulent velocity fluctuations is limited to the upper part of the bed, whereas the pressure fluctuations allow TKE to be transported to much lower bed levels.

(iv) Analysis of spectra and two point statistics revealed that the turbulence imposed by the surface flow gradually dissipates while penetrating within the porous medium, which behaves as a low pass filter. Indeed dissipation occurs faster for the small scales of turbulence than for the large ones, which instead are persistent, although weak, even at the lowest bed levels.

\section{ACKNOWLEDGMENTS}

This work was funded by the United Kingdom Engineering and Physical Sciences Research Council under Grant No. GR/R51865/01. The authors are grateful to two anonymous reviewers for insightful comments and useful suggestions.

${ }^{1}$ A. F. E. Zagni and K. V. H. Smith, "Channel flow over permeable beds of graded spheres,” J. Hydr. Div. 102, 207 (1976).

${ }^{2}$ H. J. Zippe and W. H. Graf, “Turbulent boundary-layer flow over permeable and non-permeable rough surfaces," J. Hydr. Res. 21, 51 (1983).

${ }^{3}$ W. P. Breugem, B. J. Boersma, and R. E. Uittenbogaard, "The influence of wall permeability on turbulent channel flow," J. Fluid Mech. 562, 35 (2006).

${ }^{4}$ G. S. Beavers and D. D. Joseph, "Boundary conditions at a naturally permeable wall," J. Fluid Mech. 30, 197 (1967).

${ }^{5}$ R. E. Larson and J. J. Higdon, "Microscopic flow near the surface of two-dimensional porous media. Part 1. Axial flow," J. Fluid Mech. 166, 449 (1986).

${ }^{6} \mathrm{~K}$. Vafai and R. Thiyagaraja, "Analysis of flow and heat transfer at the interface region of a porous medium,” Int. J. Heat Mass Transfer 30, 1391 (1987).

${ }^{7}$ M. Sahraoui and M. Kaviany, "Slip and no-slip velocity boundary conditions at the interface of porous, plain media," Int. J. Heat Mass Transfer 35, 927 (1992).

${ }^{8}$ A. J. Ochoa-Tapia and S. Whitaker, "Momentum transfer at the boundary between a porous medium and a homogeneous fluid. I: Theoretical development," Int. J. Heat Mass Transfer 38, 2635 (1995).

${ }^{9}$ A. J. Ochoa-Tapia and S. Whitaker, "Momentum transfer at the boundary between a porous medium and a homogeneous fluid. II: Comparison with experiment," Int. J. Heat Mass Transfer 38, 2647 (1995).

${ }^{10}$ C. Y. Choi and P. M. Waller, "Momentum transport mechanism for water flow over porous media,” J. Environ. Eng. 123, 792 (1997).
${ }^{11}$ D. F. James and A. M. J. Davis, "Flow at the interface of a model fibrous porous medium," J. Fluid Mech. 426, 47 (2001).

${ }^{12}$ U. Shavit, G. Bar-yosef, and R. Rosenzweig, "Modified Brinkman equation for a free flow problem at the interface of porous surfaces: The Cantor-Taylor brush configuration case," Water Resour. Res. 38, 1320, doi:10.1029/2001WR001142 (2002).

${ }^{13}$ J. F. Ruff and L. W. Gelhar, "Turbulent shear flow in porous boundary," J. Engrg. Mech. Div. 98, 975 (1972).

${ }^{14}$ Y. Shimizu, T. Tsujimoto, and H. Nakagawa, "Experiment and macroscopic modelling of flow in highly permeable porous medium under freesurface flow," J. Hydrosci. Hydr. Eng. 8, 69 (1990).

${ }^{15}$ S. Vollmer, R. Francisco de los Santos, H. Daebel, and G. Kuhn, "Microscale exchange processes between surface and subsurface water," J. Hydrol. 269, 3 (2002).

${ }^{16}$ B. A. Kironoto and W. H. Graf, "Turbulence characteristics in rough uniform open-channel flow," Proc. Inst. Civ. Eng., Waters. Maritime Energ. 106, 333 (1994)

${ }^{17}$ N. A. Horton and D. Pokrajac, "Onset of turbulence in a regular porous medium: An experimental study," Phys. Fluids 21, 045104 (2009).

${ }^{18}$ C. Manes, D. Pokrajac, and I. K. McEwan, "Double-averaged open channel flows with small relative submergence," J. Hydr. Eng. 133, 896 (2007).

${ }^{19}$ M. Raffel, C. E. Willert, and J. Kompenhans, Particle-Image Velocimetry: A Practical Guide (Springer-Verlag, Berlin, 1998).

${ }^{20}$ J. D. Smith and S. R. McLean, "Macro-turbulent structure of open channel flow over gravel beds," J. Geophys. Res. 82, 1735, doi:10.1029/ JC082i012p01735 (1977).

${ }^{21}$ Met-Flow SA, UVP monitor model UVP-XW, user guide, 2000.

${ }^{22}$ C. Manes, D. Pokrajac, I. K. McEwan, V. I. Nikora, and L. J. Campbell, "A new application of UVP: Velocity measurements within porous media,” J. Hydr. Eng. 132, 983 (2006).

${ }^{23}$ L. A. Giménez-Curto and M. A. Corniero Lera, "Oscillating turbulent flow over very rough surfaces,” J. Geophys. Res. 101, 20745, doi:10.1029/ 96JC01824 (1996).

${ }^{24}$ V. I. Nikora, D. Goring, I. K. McEwan, and G. Griffiths, "Spatiallyaveraged open-channel flow over rough bed," J. Hydr. Eng. 127, 123 (2001).

${ }^{25}$ D. Pokrajac, C. Manes, and I. K. McEwan, "Peculiar mean velocity profiles within a porous bed of an open channel," Phys. Fluids 19, 098109 (2007).

${ }^{26}$ C. Manes, D. Pokrajac, O. Coceal, and I. McEwan, "On the significance of form-induced stress in rough wall turbulent boundary layers," Acta Geophys. 56, 845 (2008).

${ }^{27}$ V. Nikora, I. McEwan, S. McLean, S. Coleman, D. Pokrajac, and R. Walters, "Double-averaging concept for rough-bed open-channel and overland flows: Theoretical background," J. Hydr. Eng. 133, 873 (2007).

${ }^{28}$ V. Nikora, S. McLean, S. Coleman, D. Pokrajac, I. McEwan, L. Campbell, J. Aberle, D. Clunie, and K. Koll, "Double-averaging concept for roughbed open-channel and overland flows: Applications," J. Hyd. Eng. 133, 884 (2007)

${ }^{29}$ I. Nezu and H. Nakagawa, in Turbulence in Open Channel Flow, edited by A. Balkema (IAHR, Rotterdam, 1993).

${ }^{30}$ V. Nikora, K. Koll, I. K. McEwan, S. McLean, and A. Dittrich, "Velocity distribution in the roughness layer of rough bed flows," J. Hyd. Eng. 130, 1036 (2004).

${ }^{31}$ H. Nakagawa and I. Nezu, "Structure of space-time correlations of bursting phenomena in an open channel flow," J. Fluid Mech. 104, 1 (1981).

${ }^{32}$ A. B. Shvidchenko and G. Pender, "Macro-turbulent structure of open channel flow over gravel beds," Water Resour. Res. 37, 709, doi:10.1029/ 2000WR900280 (2001)

${ }^{33}$ A. G. Roy, T. Buffin-Belanger, H. Lamarre, and A. D. Kirkbride, "Size, shape and dynamics of large-scale turbulent flow structures in a gravel-bed river," J. Fluid Mech. 500, 1 (2004).

${ }^{34}$ I. Marusic and N. Hutchins, "Study of the log layer structure in wall turbulence over a very large range of Reynolds number," Flow, Turbul. Combus. 81, 115 (2008). 